\title{
Tracheobronchopathia osteochondroplastica discovered during tracheal intubation for general anesthesia
}

\author{
Chan Noh', Yong Sup Shin ${ }^{1,2}$, Seok Hwa Yoon ${ }^{1,2}$, Seok Hwan Hong', Choon Ho Jung', \\ Chae Seong Lim ${ }^{1,2}$ \\ ${ }^{1}$ Department of Anesthesiology and Pain Medicine, Chungnam National University Hospital, ${ }^{2}$ Department of Anesthesiology and Pain \\ Medicine, Chungnam National University School of Medicine, Daejeon, Korea
}

Received March 26, 2018

Revised April 10, 2018

Accepted April 10, 2018

Corresponding author

Chae Seong Lim

Department of Anesthesiology and

Pain Medicine, Chungnam National

University Hospital, Chungnam

National University School of

Medicine, 282 Munhwa-ro, Jung-

gu, Daejeon 35015, Korea

Tel: $+82-42-280-7840$

Fax: +82-42-280-7968

E-mail: limtwo2@gmail.com

ORCID:

https://orcid.org/0000-0002-2356-8999

\begin{abstract}
Tracheobonchopathia osteochondroplastica (TO) is a rare disorder, where there is benign dysplasia of the trachea and occasionally large bronchi. We report the case of a 55-year-old woman who was discovered to have TO at the time of a difficult intubation. This case was also unusual because the patient had presented no previous symptoms despite the presence of extensive endotracheal lesions. The final diagnosis of TO was made after subsequent bronchoscopy in the post-operative period.
\end{abstract}

Keywords: Bronchoscopy; Anesthesia; Intratracheal intubation

\section{INTRODUCTION}

Tracheobronchopathia osteochondroplastica (TO) disorder is characterized as multiple, irregular, bony or cartilaginous nodules covering the normal epithelial tracheal submucosa. TO was first described by Wilks in an autopsy of a 38-year-old man who died from tuberculosis in 1857 [1]. Although nodules can sometimes reach the main bronchus, they rarely reach the larynx. Because this disease is usually benign, clinical signs are rarely specific; nevertheless, dry cough, dyspnea, recurrent respiratory infection, and occasional blood exudation have been noted. A case of difficult intubation, in whom the patient was diagnosed with TO postoperatively, is reported.

\section{CASE}

Informed written consent was obtained from the patient for publication of this case report. A 55-year-old woman, a non-smoker, underwent laparoscopic surgery for benign cysts of the ovary. She had no relevant medical history or respiratory symptoms. Anesthesia was induced using propofol and rocuronium. Oxygen saturation during respiration was $99 \%$. After introduction of anesthesia, the patient was hemodynamically stable, and oxygen saturation during ventilation

This is an Open Access article distributed under the terms of the Creative Commons Attribution Non-Commercial License (http://creativecommons.org/licenses/by-nc/4.0) which permits unrestricted noncommercial use, distribution, and reproduction in any medium, provided the original work is properly cited. Copyright $@$ Medical Biological Science and Engineering. 

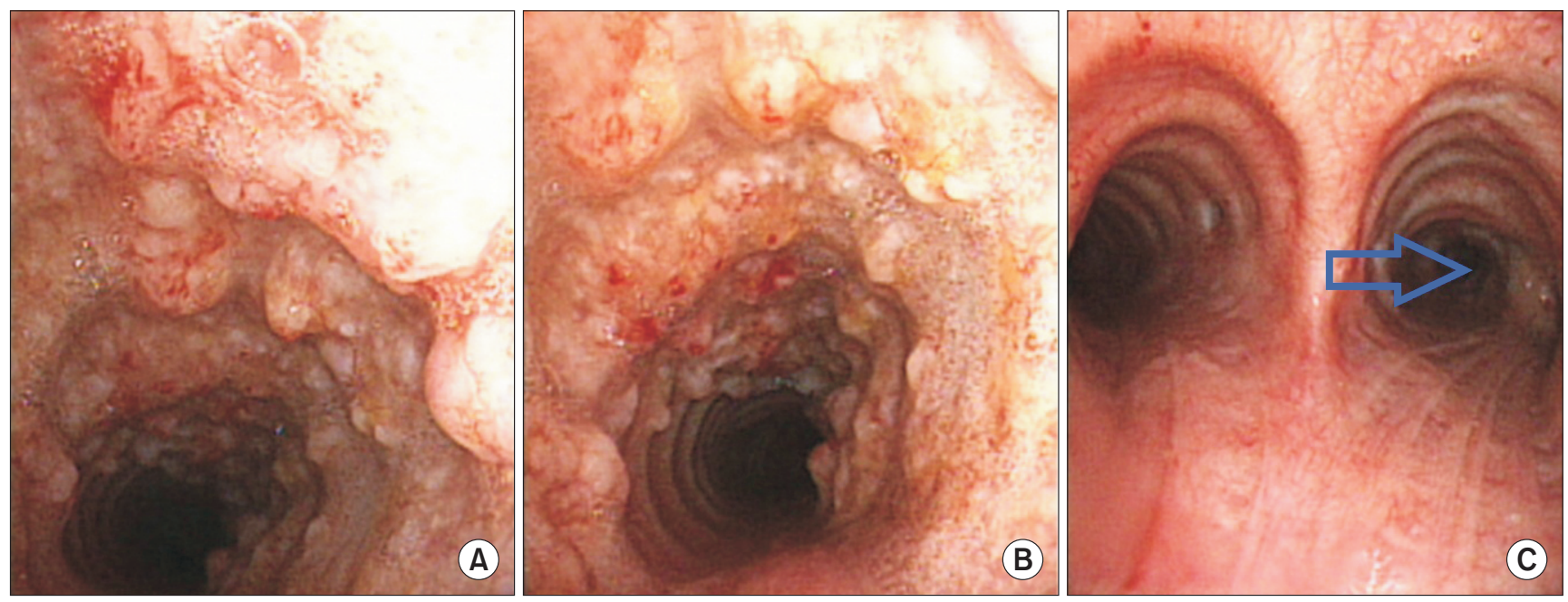

Fig. 1. Bronchoscopy view: the numerous bony and cartilaginous nodules are present at the anterior and lateral aspects of the tracheal wall. Subglottic region (A), trachea wall $(B)$, and carina (C). A blue arrow indicates mild remote region of right middle lobe entrance.

with $100 \%$ oxygen was $100 \%$. Mask ventilation was satisfactory and without resistance. In laryngoscopy, the larynx was readily visualized; however, a cuffed tracheal tube (size 7.5 $\mathrm{mm}$ ) encountered resistance below the glottis and was subsequently removed. Another cuffed tracheal tube (size $7.0 \mathrm{~mm}$ ) was inserted using a Glidescope video laryngoscope (Glidescope $^{\circledR}$; Saturn Biomedical Systems Inc., Burnaby, BC, Canada). The tube was inserted to a depth of only $19 \mathrm{~cm}$. In normal females, the average tube depth is $21 \mathrm{~cm}$; however, due to resistance, it was not possible to insert it more than $19 \mathrm{~cm}$. Fiber bronchoscopy was performed through a tracheal tube after surgery. Many white, bony structures were observed. After the patient recovered from surgery, she was introduced to a respiratory physician, who performed bronchoscopic examination and attempted biopsy of the lesion. The lesion, however, was too hard to be grasped with forceps; consequently, biopsy was abandoned. Bronchoscopy examination resulted in a diagnosis of TO. The protruding gravel-like lesion was present from the upper trachea to the entrance of the right middle lobe (Fig. 1). After bronchoscopy, the patient was introduced to an otolaryngologist for surgical biopsy; however, she refused the procedure and was discharged without further treatment.

\section{DISCUSSION}

TO is a rare and benign pathology characterized by the presence of bony and cartilaginous nodules in the trachea and bronchial mucosa. The prevalence of TO has been
Table 1. Incidence of tracheobronchopathia osteochondroplastica (TO) reported in previous publications

\begin{tabular}{lcc}
\hline \multicolumn{1}{c}{ Author } & Bronchoscopy & TO \\
\hline Pérez-Rodriguez et al. [2] & 8,700 & $8(0.09)$ \\
Prakash [3] & 772 & $1(0.12)$ \\
Lundgren and Stjernberg [4] & 2,180 & $9(0.41)$ \\
Sen and Walsh [5] & 1,500 & $2(0.13)$ \\
Tatar et al. [6] & 31,841 & $4(0.01)$ \\
Present study & 19,197 & $4(0.02)$ \\
\hline
\end{tabular}

Values are presented as number only or number (\%).

reported to be $0.01 \%$ to $4.2 \%$ in patients undergoing bronchoscopy (Table 1) [2-6]. TO is found at similar rates in men and women, and generally diagnosed at $\geq 50$ years of age [7]; however, young adults and children may also be affected [8]. Although the cause of the condition is unknown, several theories have been proposed. Sakula [७] advocated the theory that bronchiectasis is a form of primary localized amyloidosis of the lower respiratory tract that has undergone ossification. Weiss [10] reported that TO is associated with amyloidosis. Several reports have described the association between TO and atrophic rhinitis [11,12]. TO produces no characteristic symptoms or signs indicative of asthma or chronic bronchitis. This disease manifests as chronic cough and wheezing, often leading to a misdiagnosis of asthma or chronic bronchitis. Bronchoscopy is the only technique able to reliably establish a diagnosis of TO. Because TO typically presents as an obstacle, it is unnecessary to diagnose TO via biopsy of an airway luminal nodule. In fact, due to the stiffness of the lesion, it is 
usually difficult to obtain a biopsy of lesions protruding into the respiratory tract [13], similar to our case.

It is important to recognize that imaging examination rarely leads to TO diagnosis. Standard chest X-rays are usually normal and not useful for diagnosis. In our case, preoperative simple posteroanterior chest X-ray yielded normal findings. It is impossible to remove all nodules or prevent recurrence; therefore, treatment of only symptomatic TO is necessary. For patients with cough, wheezing and/or respiratory infections, conservative treatment, such as maintenance of airway humidity, minimization of airway irritation and rapid treatment of infectious diseases, is recommended. Morbidity appears to be proportional to airway rigidity, the degree of obstruction, and underlying health status [13]. If this rare condition is found during intubation, the extent of disease and the urgency of the surgery may influence the decision as to whether to continue the operation. A highly severe case of TO was previously reported, in which a microlaryngoscope tube (size, $4.0 \mathrm{~mm}$ ) eventually passed through a bougie placed through a rigid bronchoscope [14]. After the lesion was removed using a carbon-dioxide laser, the patient was intubated the next day. Although lesions were extensive, they were superficial. In our case, interference components were minimal. Unfortunately, we had to exclude supraglottic airway because of the Trendelenburg position and pneumoperitoneum for laparoscopic surgery. If the anticipated procedure has a low risk for pulmonary aspiration, the supraglottic airway is a good choice [15]. The consideration of anesthetics for this condition is similar to tracheal stenosis due to other causes if symptoms are severe. If TO is suspected, intubation should be followed by bronchoscopy and tracheal biopsy. If bronchoscopy is impossible due to subglottic narrowing, and biopsy is difficult due to calcification, computed tomography should be performed.

In our case, the patient exhibited no symptoms despite the presence of extensive endotracheal lesions. Anesthesiologists should be aware that $\mathrm{TO}$ is one of many causes of unexpected and difficult intubation. It is advantageous, however, that the management of such cases is similar to those for other difficult airways.

\section{CONFLICT OF INTEREST}

No potential conflict of interest relevant to this article was reported.

\section{REFERENCES}

1. Wilks S. Ossific deposit on the larynx, trachea and bronchi. Trans Pathol Soc Lond 1857;8:88.

2. Pérez-Rodriguez E, Núñez N, Alvarado C, Golpe A, Casanova C, Zapatero J, et al. Diagnosis of tracheopathia osteochondroplastica. Chest 1990;97:763.

3. Prakash UBS. Uncommon causes of cough: ACCP evidencebased clinical practice guidelines. Chest 2006;129(1 Suppl): 206S-19S.

4. Lundgren R, Stjernberg NL. Tracheobronchopathia osteochondroplastica. A clinical bronchoscopic and spirometric study. Chest 1981;80:706-9.

5. Sen RP, Walsh TE. Fiberoptic bronchoscopy for refractory cough. Chest 1991;99:33-5.

6. Tatar D, Senol G, Demir A, Polat G. Tracheobronchopathia osteochondroplastica: four cases. Chin Med J (Engl) 2012;125: 2942-4.

7. Smith DC, Pillai R, Gillbe CE. Tracheopathia osteochondroplastica. A cause of unexpected difficulty in tracheal intubation. Anaesthesia 1987;42:536-8.

8. Simsek PO, Ozcelik U, Demirkazik F, Unal OF, Orhan D, Aslan AT, et al. Tracheobronchopathia osteochondroplastica in a 9-year-old girl. Pediatr Pulmonol 2006;41:95-7.

9. Sakula A. Tracheobronchopathia osteoplastica: its relationship to primary tracheobronchial amyloidosis. Thorax 1968;23:10510.

10. Weiss L. Isolated multiple nodular pulmonary amyloidosis. Am J Clin Pathol 1960;33:318-29.

11. Vaheri E, Vaheri E. Tracheopathia osteoplastica. Acta Otolaryngol 1967;64:251-5.

12. Härmä RA, Suurkari S. Tracheopathia chondro-osteoplastica. A clinical study of thirty cases. Acta Otolaryngol 1977;84:118-23.

13. Prakash UB. Tracheobronchopathia osteochondroplastica. Semin Respir Crit Care Med 2002;23:167-75.

14. Birzgalis AR, Farrington WT, O’Keefe L, Shaw J. Localized tracheopathia osteoplastica of the subglottis. J Laryngol Otol 1993;107:352-3.

15. Ishii H, Fujihara H, Ataka T, Baba H, Yamakura T, Tobita T, et al. Successful use of laryngeal mask airway for a patient with tracheal stenosis with tracheobronchopathia osteochondroplastica. Anesth Analg 2002;95:781-2. 